\title{
Cooling by heating: Restoration of the third law of thermodynamics
}

\author{
V. B. Sørdal, ${ }^{1, *}$ J. Bergli, ${ }^{1}$ and Y. M. Galperin ${ }^{1,2}$ \\ ${ }^{1}$ Department of Physics, University of Oslo, P.O. Box 1048 Blinderm, 0316 Oslo, Norway \\ ${ }^{2}$ Ioffe Institute, 26 Politekhnicheskaya, St. Petersburg 194021, Russian Federation
}

(Received 20 November 2015; published 2 March 2016)

\begin{abstract}
We have made a simple and natural modification of a recent quantum refrigerator model presented by Cleuren et al. [Phys. Rev. Lett. 108, 120603 (2012)]. The original model consist of two metal leads acting as heat baths and a set of quantum dots that allow for electron transport between the baths. It was shown to violate the dynamic third law of thermodynamics (the unattainability principle, which states that cooling to absolute zero in finite time is impossible). By taking into consideration the finite energy level spacing $\Delta$, in metals we restore the third law while keeping all of the original model's thermodynamic properties intact down to the limit of $k_{B} T \sim \Delta$, where the cooling rate is quenched. The spacing $\Delta$ depends on the confinement of the electrons in the lead and therefore, according to our result larger samples (with smaller level spacing), could be cooled efficiently to lower absolute temperatures than smaller ones. However, a large lead makes the assumption of instant equilibration of electrons implausible; in reality one would only cool a small part of the sample and we would have a nonequilibrium situation. This property is expected to be model independent and raises the question whether we can find an optimal size for the lead that is to be cooled.
\end{abstract}

DOI: 10.1103/PhysRevE.93.032102

\section{INTRODUCTION}

Quantum refrigerators are solid-state devices with huge potential benefits in technology. With no moving parts and of microscopic size, they could easily be integrated into existing technology, such as cellphones and computers, to enhance their performance by utilizing the waste heat energy they produce. As always, the technological frontier is supported by a backbone of theoretical framework, which in recent years has seen many advancements (see, e.g., [1-6]). In addition to the technological possibilities they present, quantum refrigerators are excellent tools for providing insight into the unique features of open quantum systems. For a review of stochastic thermodynamics and the formalism used to treat quantum refrigerators see, e.g., [7,8].

The quantum absorption refrigerator is a version of these general machines, based on producing a steady-state heat flow from a cold to a hot reservoir, driven by absorption from an external heat reservoir. A key tool to understand the operation of these refrigerators, when approaching the limiting temperature of absolute zero, is the laws of thermodynamics. In this article we study one such device that appears to violate the dynamic version of the third law of thermodynamics (the unattainability principle), which states that one cannot cool a system to absolute zero in a finite amount of time. A recent publication by Cleuren et al. [9] presented a novel model based on two electronic baths coupled together via a system of quantum dots and driven by an external photon source. The article generated some controversy due to its apparent violation of the unattainability principle, and several authors [10-13] proposed explanations for this violation. However, we find that the discussion was without conclusion, and we will discuss this later in the article.

We will begin by giving a brief presentation of the quantum refrigerator model, as introduced by Cleuren et al. [9], and

\footnotetext{
*v.b.sordal@fys.uio.no
}

its thermodynamic properties. Then we will summarize and comment on the discussion that followed. Finally, we will present a simple modification, based only on the fact that the energy levels of metals are discrete when treated quantum mechanically, which becomes important at temperatures $T \lesssim$ $\Delta$, where $\Delta$ is the level spacing. (We measure temperature in energy units, setting the Boltzmann constant $k_{B}=1$.) Our modification upholds the third law while it simultaneously reproduces the results from the original model down to the limit of $T \sim \Delta$. In essence, we want to make the point that the validity of the unattainability principle is only guaranteed when applied to a quantum description of a system and that the most important quantum effect to consider in relation to this law is the discretization of energy states.

\section{A. Model}

The quantum refrigerator model proposed in [9] is shown schematically in real space in Fig. 1 and in energy space in Fig. 2. Here we briefly explain its operating protocol. It consists of two metal leads and four quantum dots; the large and hot lead with temperature $T_{L}$ is coupled to the small cold lead with temperature $T_{R}$, via the set of quantum dots. We assume that each quantum dot is highly confined and is thus associated with a single energy level, since the other levels are far outside the energy range of the system. These four levels are marked in Fig. 2. The quantum dots form two channels, as illustrated in Fig. 1, where the energy levels $\epsilon_{2}\left(\epsilon_{1}\right)$ and $\epsilon_{2}+\epsilon_{g}\left(\epsilon_{1}-\epsilon_{g}\right)$ are coupled together in channel 2 (channel 1). The two channels are spatially separated, therefore we can safely ignore any Coulomb interaction between the electrons in channels 1 and 2. The basic idea is to move cold electrons (i.e., with energy less than $\mu$ ) from the hot lead into the cold lead via channel 1 while simultaneously moving hot electrons (energy greater than the chemical potential $\mu$ ) from the cold lead to the hot lead via channel 2. This transport of electrons will thus cool the right lead by injecting cold and extracting hot electrons. Naturally the transport will also heat up the left lead, but since we assume 


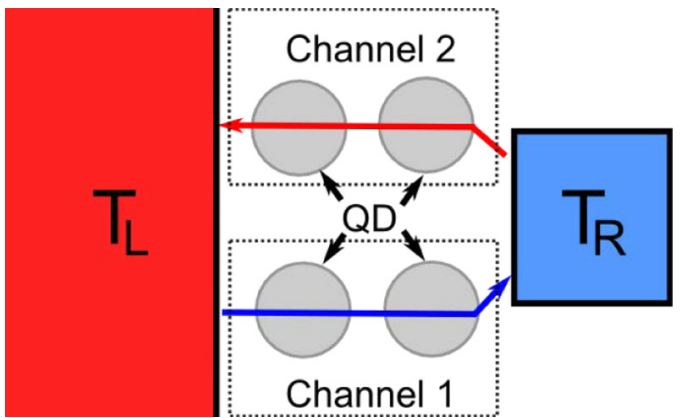

FIG. 1. Schematic of the model shown in real space. A small piece of metal with temperature $T_{R}$ is coupled to a larger piece with temperature $T_{L}>T_{R}$. Four quantum dots form two channels for electron transport between the metals. The arrows indicate the desired direction of the net particle current to achieve cooling of the right metal lead. The distance between the two channels is too large for any Coulomb interaction to take place between them.

that it is a large piece of metal with a high heat capacity, the heat absorbed will not result in a measurable change in $T_{L}$. We can obtain the desired particle flow direction by coupling the quantum dot system to a bosonic bath that induces transitions between the quantum dots of each pair, i.e., between $\epsilon_{1}$ and $\epsilon_{1}-\epsilon_{g}$ in channel 1 and between $\epsilon_{2}$ and $\epsilon_{2}+\epsilon_{g}$ in channel 2 . The bosonic bath can be photons from an external source and/or phonons from the device. In this discussion we will consider it to be a photon bath with temperature $T_{S}$. In Ref. [9] the photon bath is taken to be the sun with a temperature $T_{S} \simeq 6000 \mathrm{~K}$ and we will follow this in the sense that we will assume that it is the largest energy scale in the system. In any case the transition rates between the quantum dots are proportional to the probability of finding a boson with energy equal to the energy difference between the two quantum dot levels, which is given by the Planck distribution $n(E)$. The

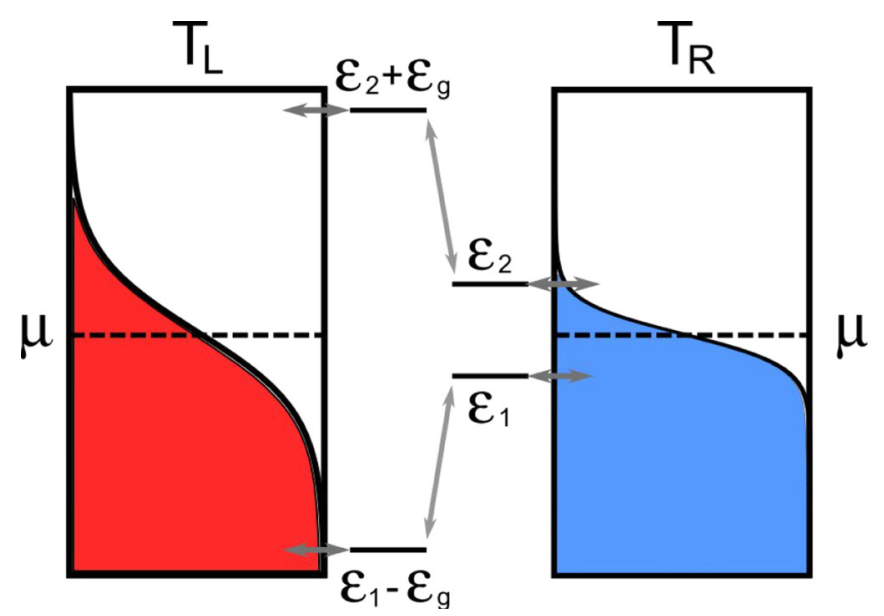

FIG. 2. A hot metal lead $T_{L}$ is coupled to a cold one $T_{R}$ via two spatially separated pairs of quantum dots, which form two channels for electron transport between the leads. We consider the case where $\mu_{L}=\mu_{R}=\mu$ and the energy levels of the quantum dots are symmetric about the chemical potential $\left(\epsilon_{2}-\mu=\mu-\epsilon_{1} \rightarrow\right.$ $\left.\epsilon_{1}=-\epsilon_{2}\right)$. The schematic is adapted from [9]. rates are thus given by

$$
k_{\uparrow}^{\epsilon_{g}}=\frac{\Gamma_{s}}{e^{\epsilon_{g} / T_{S}}-1}, \quad k_{\downarrow}^{\epsilon_{g}}=\frac{\Gamma_{s}}{1-e^{-\epsilon_{g} / T_{S}}} .
$$

Here $k^{\uparrow}$ and $k^{\downarrow}$ are the rates for upward and downward transitions in energy, respectively. The difference between them is that $k^{\downarrow}$ contains an additional term for spontaneous emission.

The transition rate for electron transfer from the metal to an empty quantum dot level is proportional to the probability of finding an electron in the same energy level in the metal, which is given by the Fermi-Dirac distribution $f(E)$. For the inverse transition to take place there has to be an available energy level in the metal, which has a probability proportional to $1-f(E)$. Thus the transition rates between the quantum dot and metal are

$$
k_{l \rightarrow d}^{E}=\frac{\Gamma}{e^{(E-\mu) / T}+1}, \quad k_{d \rightarrow l}^{E}=\frac{\Gamma}{e^{(\mu-E) / T}+1} .
$$

For transitions involving the right lead the temperature $T=$ $T_{R}$, while for the left lead $T=T_{L}$. Notice that in general $\Gamma \neq \Gamma_{s}$. These are the constants that set the time scale of the transitions and depends on the specific details of the device.

As in Ref. [9], we will considering the strongly coupled case where the energies of the quantum dots are symmetric about the chemical potential $\left(\epsilon_{2}-\mu=\mu-\epsilon_{1}\right)$. We can therefore choose to measure all energies relative to $\mu=0$ and combine the two parameters $\epsilon_{2}=-\epsilon_{1}=\epsilon$.

We can now introduce three distinct occupation probabilities per channel. Since the two quantum dots in the same channel are close to each other in space we assume that the Coulomb repulsion between electrons prevents simultaneous occupation of the right and left quantum dots. For channel 1 we then have the probabilities $P_{L}^{(1)}, P_{R}^{(1)}$, and $P_{0}^{(1)}$, which represent the probability of finding an electron in the left quantum dot with energy $-\left(\epsilon+\epsilon_{g}\right)$, in the left quantum dot with energy $-\epsilon$, and in neither quantum dot, respectively. A master equation describing the time evolution of the occupation probabilities in channel 1 can thus be formulated

$$
\dot{\mathbf{P}}^{(1)}=\hat{M}^{(1)} \mathbf{P}^{(1)}, \quad \mathbf{P}^{(1)} \equiv\left[\begin{array}{c}
P_{0}^{(1)} \\
P_{L}^{(1)} \\
P_{R}^{(1)}
\end{array}\right],
$$

where the transition matrix $M^{(1)}$ is given by

$$
\begin{aligned}
& M^{(1)} \\
& =\left[\begin{array}{ccc}
-k_{l \rightarrow d}^{-\left(\epsilon+\epsilon_{g}\right)}-k_{l \rightarrow d}^{-\epsilon} & k_{d \rightarrow l}^{-\left(\epsilon+\epsilon_{g}\right)} & k_{d \rightarrow l}^{-\epsilon} \\
k_{l \rightarrow d}^{-\left(\epsilon+\epsilon_{g}\right)} & -k_{d \rightarrow l}^{-\left(\epsilon+\epsilon_{g}\right)}-k_{\uparrow}^{\epsilon_{g}} & k_{\downarrow}^{\epsilon_{g}} \\
k_{l \rightarrow d}^{-\epsilon} & k_{\uparrow}^{\epsilon_{g}} & -k_{d \rightarrow l}^{-\epsilon}-k_{\downarrow}^{\epsilon_{g}}
\end{array}\right]
\end{aligned}
$$

We are interested in the steady state of the system, where the probabilities do not change as a function of time. To find this state we set $\dot{\mathbf{P}}^{(1)}=\mathbf{0}$ and solve Eq. (3). By doing this we obtain the steady-state probability vector $\mathbf{P}^{(1)}\left(\epsilon, \epsilon_{g}, T_{R}, T_{L}\right)$, where we consider $\Gamma, \Gamma_{s}$, and $T_{S}$ as constants. A similar procedure gives us the steady-state probability vector for channel 2 as well. 
The particle current between the right dot in the lower level and the cold lead can be written as

$$
J^{(1)}=P_{R}^{(1)} k_{d \rightarrow l}^{-\epsilon}-P_{0}^{(1)} k_{l \rightarrow d}^{-\epsilon}
$$

and the current through the upper level is

$$
J^{(2)}=P_{R}^{(2)} k_{d \rightarrow l}^{\epsilon}-P_{0}^{(2)} k_{l \rightarrow d}^{\epsilon} .
$$

The cooling power, i.e., the heat transported out of the right lead per unit time, can now be defined as

$$
\dot{Q}_{R}=(-\epsilon-\mu)\left(-J^{(1)}\right)+(\epsilon-\mu)\left(-J^{(2)}\right) .
$$

Since the energy levels are symmetric about $\mu$, we can set $\mu=0$ and we obtain the cooling power for the refrigerator model

$$
\dot{Q}_{R}=\epsilon\left(J^{(1)}-J^{(2)}\right) .
$$

Optimized cooling is attained by varying $\epsilon\left(T_{R}\right)$ and $\epsilon_{g}\left(T_{R}\right)$ as a function of $T_{R}$ (when $T_{S}$ and $T_{L}$ are kept constant). It can be shown (see Ref. [9] for details) that the cooling power in the limit of low $T_{R}$ is given by

$$
\lim _{T_{R} \rightarrow 0} \dot{Q}_{R} \propto T_{R}
$$

When working at an energy scale where $\epsilon_{g} \ll T_{S}$ we have $k^{\uparrow} \simeq k^{\downarrow}$. In this situation we can get a better understanding of the system and when cooling will occur by considering the transitions in channel 2 . There the energy levels are situated above $\mu$ and we have

$$
0<f(E)<1 / 2, \quad 1 / 2<1-f(E)<1 .
$$

Therefore, the rate from lead to dot will always be less than the rate from dot to lead $k_{l \rightarrow d}^{E}<k_{d \rightarrow l}^{E}$ for a given energy $E$. The requirement for cooling to take place in this situation is that $f\left(\epsilon+\epsilon_{g}, T_{L}\right)<f\left(\epsilon, T_{R}\right)$, i.e., we require $\left(\epsilon+\epsilon_{g}\right) / T_{L}>$ $\epsilon / T_{R}$. We then have

$$
\left.\begin{array}{l}
k_{d \rightarrow l}^{\epsilon+\epsilon_{g}}>k_{d \rightarrow l}^{\epsilon} \\
k_{l \rightarrow d}^{\epsilon+\epsilon_{g}}<k_{l \rightarrow d}^{\epsilon} \\
k_{l \rightarrow d}^{E d}<k_{d \rightarrow l}^{E}
\end{array}\right\} \Rightarrow k_{d \rightarrow l}^{\epsilon+\epsilon_{g}}>k_{d \rightarrow l}^{\epsilon}>k_{l \rightarrow d}^{\epsilon}>k_{l \rightarrow d}^{\epsilon+\epsilon_{g}} .
$$

When $k^{\uparrow} \simeq k^{\downarrow}$ we know that the occupation probability $P_{L}^{(2)} \simeq$ $P_{R}^{(2)}=P$ and thus $P_{0}^{(2)}=1-2 P$. Using the inequalities shown in Eq. (9), we now consider two different states of the system. First assume that there is an electron in the quantum dot system; it can exit into either the left lead or the right lead, where the currents are $k_{d \rightarrow l}^{\epsilon+\epsilon_{g}} P_{L}^{(2)}$ and $k_{d \rightarrow l}^{\epsilon} P_{R}^{(2)}$, respectively. The difference is

$$
P\left(k_{d \rightarrow l}^{\epsilon+\epsilon_{g}}-k_{d \rightarrow l}^{\epsilon}\right)>0,
$$

which tells us that it is more likely for the electron to exit into the left lead. Next we assume that the quantum dot system is unoccupied; an electron can enter from the left lead or the right lead, with currents $k_{l \rightarrow d}^{\epsilon+\epsilon_{g}} P_{0}^{(2)}$ and $k_{l \rightarrow d}^{\epsilon} P_{0}^{(2)}$, respectively. The difference is now

$$
(1-2 P)\left(k_{l \rightarrow d}^{\epsilon+\epsilon_{g}}-k_{l \rightarrow d}^{\epsilon}\right)<0,
$$

indicating that it is more likely that an electron enters from the right lead. Above the chemical potential, electrons entering from the right lead and exiting into the left lead correspond to a net cooling of the right lead, which is our desired effect.
A similar analysis can be done for channel 1 , where the corresponding result of net transport from the left to the right lead is obtained.

In summary, one obtains optimal cooling of the right lead by varying the energy levels $\epsilon_{2}=-\epsilon_{1}=\epsilon$ as a function of $T_{R}$ and their optimal position is determined by a balance between the transport rate (higher closer to $\mu$ ) and heat removed per transition (higher far from $\mu$ ), and the additional requirement that $f\left(\epsilon+\epsilon_{g}, T_{L}\right)<f\left(\epsilon, T_{R}\right)$.

\section{B. Unattainability principle}

The unattainability principle states that one cannot cool a system to absolute zero in a finite amount of time [14]. A system with heat capacity $C_{V}=d Q / d T$ and cooling power $\dot{Q}=d Q / d t$ has a cooling rate given by

$$
\frac{d T}{d t}=\frac{\dot{Q}}{C_{V}} .
$$

If we assume that $C_{V}$ and $\dot{Q}$ scale with temperature to the power of $\kappa$ and $\lambda$, respectively, we have

$$
\frac{d T}{d t} \propto T^{\lambda-\kappa} .
$$

For $\alpha \equiv \lambda-\kappa<1$ the unattainability principle is violated [10] and cooling to absolute zero is possible in a finite time. By inspecting Eq. (8) we find that $\lambda=1$. The heat capacity of the metal lead as $T_{R} \rightarrow 0$ is dominated by the electronic heat capacity, which is proportional to the temperature $C_{V} \propto T_{R}$ (see, e.g., Ref. [15]), and therefore $\kappa=1$. The end result is that $\dot{T}_{R} \propto T^{0}$, in violation of the unattainability principle.

\section{Comments}

Levy et al. [10] were the first to point out that because the refrigerator presented in [9] has a cooling power of $\dot{Q} \propto T_{R}$ and a heat capacity of $C_{V} \propto T_{R}$ in the limit of $T_{R} \rightarrow 0 \mathrm{~K}$, its cooling rate is given by

$$
\frac{d T(t)}{d t}=\frac{\dot{Q}}{C_{V}} \propto T_{R}^{0}=\text { const. }
$$

That enables cooling to absolute zero in a finite amount of time. In the original model proposed by Cleuren et al. the quantum dot system consisted of only two quantum dots, with the levels $\epsilon_{1}\left(\epsilon_{1}-\epsilon_{g}\right)$ and $\epsilon_{2}\left(\epsilon_{2}+\epsilon_{g}\right)$ being two adjacent levels within the right (left) quantum dot. Levy et al. suggest that the violation of the third law may be due to the neglect of internal transitions within a single dot. This suggestion was refuted by Cleuren et al. [11], who stated that the model could also be constructed using two pairs of spatially separated quantum dots, as we have done here. Their own explanation for the violation was that the quantum master equation they utilized does not take into account coherent effects and the broadening of the linewidth of the quantum dot energy levels was ignored. Both of these effects becomes important in the low-temperature limit.

Allahverdyan et al. [12] suggested that the violation occurs since the weak-coupling master equation used by Cleuren $\mathrm{et} \mathrm{al}$. is limited at low temperatures. They state that one can justify taking the limit $T_{R} \rightarrow 0$ for such an equation only while 
simultaneously reducing the coupling between the quantum dot system and heat reservoirs $\gamma \rightarrow 0$. Concrete analysis of the low-temperature behavior of the cooling power is not given.

Finally, Entin-Wohlman and Imry [13] considered a simplified version of the original model, where only a single channel contributes to the electron transfer. They assumed that boson-assisted hopping is the dominant form of electronic transport [2] (an assumption we will also make later in the article). If we remove channel 1 from our model and only consider channel 2, we obtain the same system as considered in [2]. Using Fermi's golden rule, they found that the heat current is exponentially small for $\epsilon_{2}-\mu \gg T_{R}$. They went on to state that the violation of the third law comes from allowing the levels $\epsilon_{1}$ and $\epsilon_{2}$ to approach the chemical potential linearly as a function of temperature and claimed that this is unnecessary and complicates the setup. In our opinion, the linear temperature dependence of the energy levels $\epsilon_{1}$ and $\epsilon_{2}$

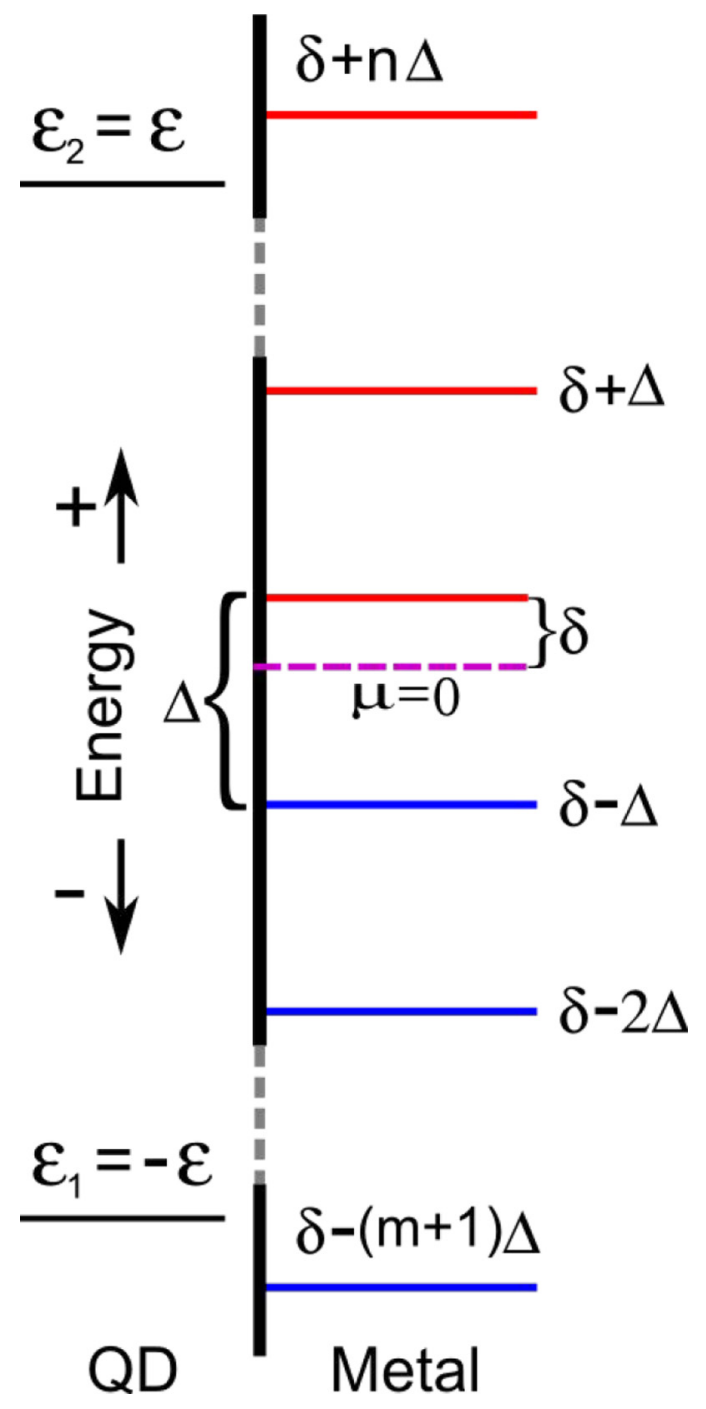

FIG. 3. The continuous states of the metal are replaced by a discrete spectrum with a constant energy spacing $\Delta$. The asymmetry between states above and below $\mu$ is modeled by the parameter $\delta$. For $\delta=\Delta / 2$ the chemical potential lies exactly in the middle of two energy levels. The $j$ th ( $i$ th) level below (above) $\mu$ is given by $\epsilon_{j}=\delta-j \Delta\left[\epsilon_{i}=\delta+(i-1) \Delta\right]$. in the quantum dots coupled to the cold lead is an essential feature; it arises from the optimization of the cooling power suggested in Ref. [9], but not implemented in Ref. [13].

\section{DISCRETIZATION OF THE MODEL}

One of the assumptions of the model proposed is that there is a continuous spectrum of energy states in the metal leads. Thus the electrons are transferred elastically between the quantum dots and the metals. We will now introduce a simple discretized modification of the original model and show that the unattainability principle will then be restored. In our model, we assume an even spacing between the energy levels. We also introduce the parameter $\delta$ to quantify the asymmetry about the chemical potential $\mu$ (see Fig. 3). If $\delta=\Delta / 2$ the energy levels are symmetrically distributed about $\mu$. As long as the quantum dot and metal energy levels do not exactly overlap, the transitions are now inelastic and require absorption or emission of phonons.

\section{A. Cooling power}

We can set up a master equation for the dynamics in channel 1, as in Eq. (3), but now for the discrete system. The rate matrix is almost identical, but since we allow for phononassisted transitions, the rates between the quantum dots and the discrete levels of the right lead are given by a sum of all possible emission and absorption transitions. We will use $\epsilon_{n}$ $\left(\epsilon_{m}\right)$ to denote the $n$th ( $m$ th) level in the metal lead, above (below) the quantum dot level $\epsilon_{1}$. We also introduce $\omega_{n}=$ $\epsilon_{n}-\epsilon_{1}$ and $\omega_{m}=\epsilon_{1}-\epsilon_{m}$ to represent the phonon frequencies associated with transitions between these levels. For transitions from the lead to the dot, $\epsilon_{n}$ and $\epsilon_{m}$ are the energies associated with emission and absorption processes, respectively, while for dot-to-lead transitions the association is opposite. The matrix elements change from $k_{d \rightarrow l}^{\epsilon_{1}} \rightarrow k_{d \rightarrow l}^{d, \epsilon_{1}}$ and $k_{l \rightarrow d}^{\epsilon_{1}} \rightarrow k_{l \rightarrow d}^{d, \epsilon_{1}}$, where we use the superscript $d$ to indicate that it is the transition rate for the discrete model. These rates are then sums of all possible emission and absorption processes and can be written as

$$
\begin{aligned}
& k_{d \rightarrow l}^{d, \epsilon_{1}}=\overbrace{\sum_{m} k_{d \rightarrow l}^{\epsilon_{m}}}^{\text {emission }}+\overbrace{\sum_{n} k_{d \rightarrow l}^{\epsilon_{n}}}^{\text {absorption }}, \\
& k_{l \rightarrow d}^{d, \epsilon_{1}}=\underbrace{\sum_{m} k_{l \rightarrow d}^{\epsilon_{m}}}_{\text {absorption }}+\underbrace{\sum_{n} k_{l \rightarrow d}^{\epsilon_{n}}}_{\text {emission }},
\end{aligned}
$$

where the emission and absorption rates are given by

$$
\begin{aligned}
k_{d \rightarrow l}^{\epsilon_{n}} & =\Gamma\left[1-f\left(\epsilon_{n}\right)\right] n\left(\omega_{n}\right) \omega_{n}^{2}, \\
k_{d \rightarrow l}^{\epsilon_{m}} & =\Gamma\left[1-f\left(\epsilon_{m}\right)\right]\left[n\left(\omega_{m}\right)+1\right] \omega_{m}^{2}, \\
k_{l \rightarrow d}^{\epsilon_{m}} & =\Gamma f\left(\epsilon_{m}\right) n\left(\omega_{m}\right) \omega_{m}^{2}, \\
k_{l \rightarrow d}^{\epsilon_{n}} & =\Gamma f\left(\epsilon_{n}\right)\left[n\left(\omega_{n}\right)+1\right] \omega_{n}^{2} .
\end{aligned}
$$

Here $n(\omega)=\left(e^{\omega / T_{R}}-1\right)^{-1}$ is the Planck distribution, which tells us the probability of finding a phonon with energy $\omega$, and $f(\epsilon)=\left(e^{\epsilon / T_{R}}+1\right)^{-1}$ is the Fermi-Dirac distribution, which tells us the probability of finding an occupied state at $\epsilon$. We assume a three-dimensional phonon density of states, thus the 
rates have to be multiplied by a $\omega^{2}$ term. We have absorbed all other constants from the density of states into the $\Gamma$ introduced earlier.

The transitions between the left quantum dot and the hot left lead, i.e., the rates involving $-\left(\epsilon+\epsilon_{g}\right)$, remain unchanged since we still consider this to be a large metal piece with a quasicontinuous energy spectrum. Again, we solve the master equation in the steady state and obtain the occupation probability vector $\mathbf{P}^{(1)}$, but now for the discrete model. With this we can find the particle currents in the channel 1 for the discrete model,

$$
J_{d}^{(1)}=P_{R}^{(1)} k_{d \rightarrow l}^{d, \epsilon_{1}}-P_{0}^{(1)} k_{l \rightarrow d}^{d, \epsilon_{1}} .
$$

Thus we can write the part of the cooling power associated with channel 1 as

$$
\begin{aligned}
\dot{Q}_{R}^{(1)}= & P_{R}^{(1)}\left(\sum_{m} k_{d \rightarrow l}^{\epsilon_{m}} \epsilon_{m}+\sum_{n} k_{d \rightarrow l}^{\epsilon_{n}} \epsilon_{n}\right) \\
& -P_{0}^{(1)}\left(\sum_{m} k_{l \rightarrow d}^{\epsilon_{m}} \epsilon_{m}+\sum_{n} k_{l \rightarrow d}^{\epsilon_{n}} \epsilon_{n}\right) .
\end{aligned}
$$

An analysis similar to that shown here can be applied to channel 2 and provide its corresponding cooling power $\dot{Q}_{R}^{(2)}$. Thus the total cooling power is written as

$$
\dot{Q}_{R}=\dot{Q}_{R}^{(1)}+\dot{Q}_{R}^{(2)} \text {. }
$$

It should be noted that in the limit of $T_{R} \rightarrow 0$ only the two levels $\delta$ and $\delta-\Delta$ will contribute to the total cooling power since all levels above $\delta$ will be unoccupied and all levels below $\delta-\Delta$ will be occupied. We can now numerically optimize Eq. (17), with respect to the two parameters $\epsilon$ and $\epsilon_{g}$, while keeping $T_{L}$ and $T_{S}$ constant. Note that $\epsilon_{m}$ and $\epsilon_{n}$ are determined from $\epsilon=-\epsilon_{1}=\epsilon_{2}$ and are not free parameters. When $\epsilon_{g} \gg \epsilon$, the optimal energy of the quantum dot levels $\pm\left(\epsilon_{g}+\epsilon\right)$ is independent of $\epsilon$ and therefore also independent of $T_{R}$ (the only influence of $T_{R}$ on those levels come via the coupling to the levels $\pm \epsilon$ ). This in turn makes the optimal cooling power $\dot{Q}_{R}$ approximately independent of $\epsilon_{g}$. Hence the only free parameter for optimization is $\epsilon\left(T_{R}\right)$.

The plot of the optimized cooling power as a function of $T_{R}$ is shown in Fig. 4. As in [9], we have to use numerics to analyze the behavior of the optimized $\dot{Q}_{R}$. For simplicity we set $\delta=\Delta / 2$ and by fitting the numerical results from Eq. (17) to the Arrhenius equation $(\ln \dot{Q}=\ln A-B / T)$, we find that the optimized cooling power as $T_{R} \rightarrow 0 \mathrm{~K}$ is given by

$$
\dot{Q}_{R} \propto e^{-\Delta / 2 T_{R}}, \quad T_{R} \rightarrow 0
$$

\section{B. Heat capacity}

The heat capacity of a Fermi gas with temperatureindependent chemical potential $\mu$ can be expressed as

$$
C_{V}=\int_{0}^{\infty} d \epsilon(\epsilon-\mu) D(\epsilon) \frac{\partial f(\epsilon)}{\partial T} .
$$

Here $D(\epsilon)$ is the density of states (which is a constant in our case) and $f(\epsilon)$ is the Fermi-Dirac distribution. When going from the continuous to the discrete description we have to exchange the integral with a sum and the continuous variable

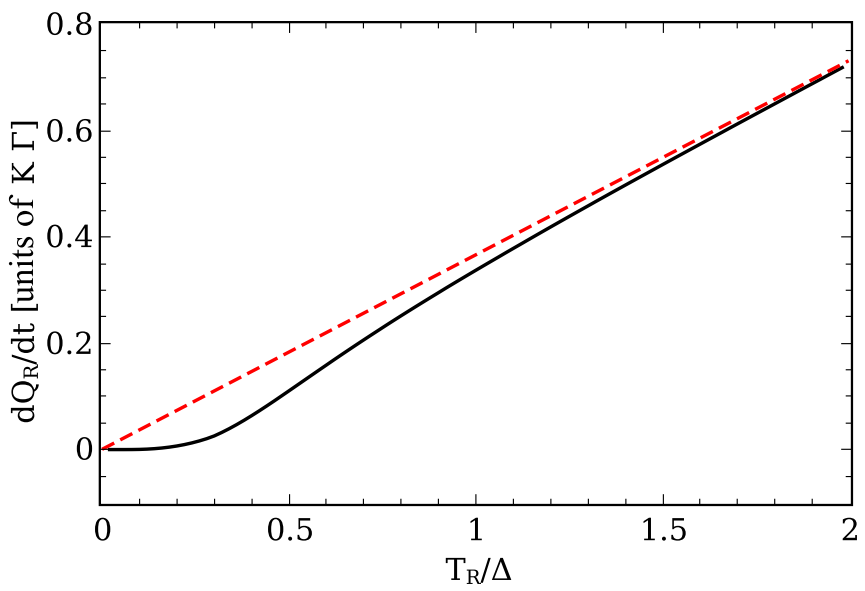

FIG. 4. Graph of the optimized cooling power $\dot{Q}_{R}$ as a function of the dimensionless variable $T_{R} / \Delta$. The dashed line is the result from the continuous model, while the solid line is the result from the discrete model. For temperatures $T_{R} \gtrsim \Delta$ the discrete model reproduces the linear cooling power of the continuous model. However, for temperatures $T_{R} \lesssim \Delta$ the cooling power changes to an exponential form. The parameters used are $\Gamma=\Gamma_{s}=1, T_{L}=20 \mathrm{~K}$, $T_{S}=6000 \mathrm{~K}, \epsilon_{g}=100 \mathrm{~K}, \Delta=1 \mathrm{~K}$, and $\delta=\Delta / 2$.

$\epsilon$ with the discretized states $n \Delta$,

$$
C_{V}=\sum_{n=0}^{\infty}\left(\frac{n \Delta-\mu}{T_{R}}\right)^{2} \frac{e^{(n \Delta-\mu) / T_{R}}}{\left(e^{(n \Delta-\mu) / T_{R}}+1\right)^{2}} .
$$

This sum can easily be determined numerically, but to gain additional insight we can consider the heat capacity for a twolevel system. As $T_{R} \rightarrow 0$ the levels $\delta$ and $\delta-\Delta$ will be the only relevant levels. We can write the grand canonical partition function for the two-level system as

$$
\Xi=1+e^{-\beta \delta}+e^{-\beta(\delta-\Delta)}+e^{-\beta(2 \delta-\Delta)}
$$

and we can find the energy via

$$
U=\frac{1}{\Xi} \sum_{i} H_{i} e^{-\beta H_{i}},
$$

where $H_{i}$ is the energy of the state $i$. From this the heat capacity can be obtained from $C_{V}=d Q / d T=d U / d T$ and we find

$$
\begin{aligned}
C_{V} & =-\frac{\Delta^{2} A+\delta^{2} B+\Delta \delta C}{T_{R}^{2}\left(1+e^{-\beta \delta}+e^{-\beta(\delta-\Delta)}+e^{-\beta(2 \delta-\Delta)}\right)^{2}}, \\
A & =e^{\beta(\Delta-3 \delta)}+e^{\beta(\Delta-\delta)}+2 e^{\beta(\Delta-2 \delta)}, \\
B & =e^{\beta(\Delta-3 \delta)}+e^{\beta(2 \Delta-3 \delta)}+e^{\beta(\Delta-\delta)}+4 e^{\beta(\Delta-2 \delta)}+e^{-\beta \delta}, \\
C & =2 e^{\beta(\Delta-3 \delta)}+2 e^{\beta(\Delta-\delta)}+4 e^{\beta(\Delta-2 \delta)} .
\end{aligned}
$$

This expression is greatly simplified at $\delta=\Delta / 2$, i.e., a symmetric distribution of energy levels above and below $\mu$. In this case we obtain

$$
C_{V}=2\left(\frac{\Delta}{2 T_{R}}\right)^{2} \frac{e^{\Delta / 2 T_{R}}}{\left(e^{\Delta / 2 T_{R}}+1\right)^{2}}
$$


and with this result, we find that in the limit of $T_{R} \rightarrow 0$ the heat capacity is

$$
C_{V}=2\left(\frac{\Delta}{2 T_{R}}\right)^{2} e^{-\Delta / 2 T_{R}}, \quad T_{R} \rightarrow 0 .
$$

Although this is only true for $\delta=\Delta / 2$, we see from the general equation for the heat capacity given in Eq. (23) that the factor of $T_{R}^{-2}$ is present for all terms and we have found numerically that the dominating exponential terms in the optimized cooling power (17) and the heat capacity (25) always cancel each other as $T_{R} \rightarrow 0$.

\section{RESULTS}

We can now find the cooling rate $d T_{R} / d t$ for the discrete system. In Fig. 4 we have plotted the cooling power $\dot{Q}_{R}$ as a function of the dimensionless variable $T_{R} / \Delta$. The solid line is the result of our numerical calculations, while the dashed line is the result from the original model [9]. We see that for $T_{R} \gtrsim \Delta$ the discrete model reproduces the results from the original model, while when $T_{R} \lesssim \Delta$ the result changes to an exponential form.

The heat capacity $C_{V}$ is shown as a function of the same dimensionless variable $T_{R} / \Delta$ in the inset in Fig. 4. Again the results from the original model are reproduced for $T_{R}>\Delta$, but when $T_{R}<\Delta / 2$ a Schottky-like feature appears, indicating that only the two levels closest to $\mu=0$ are participating in the dynamics.

As we discussed earlier, if we can write the cooling rate in a form like in Eq. (11), we require that $\alpha=\lambda-\kappa \geqslant 1$. In the original model with a continuous energy spectrum in the right metal lead, it was found that $\alpha=0$. By numerically calculating the expressions given in Eqs. (17) and (20), we find that the cooling rate is given by

$$
\frac{d T_{R}}{d t} \propto \frac{\dot{Q}_{R}}{C_{V}} \propto T_{R}^{2}, \quad T_{R} \rightarrow 0 .
$$

We obtain $\alpha=2$, which implies that cooling to absolute zero is impossible in a finite amount of time, and the discrete model is thus consistent with the unattainability principle. The result is shown in Fig. 5, where we have plotted $d T_{R} / d t$ as a function of $T_{R} / \Delta$. Also here the result from the discrete model (solid line) reproduces the result from the original model (dashed line) for $T_{R} \gtrsim \Delta$, but once $T_{R} \lesssim \Delta$ it differs.

Although the results from Eqs. (18) and (25) are only valid for $\delta=\Delta / 2$, we find numerically that the dominant exponential term in $\dot{Q}_{R}^{\text {tot }}$ always cancels with the one in $C_{V}$. The function $\frac{\dot{T}_{R}}{T_{R}^{2}}$ always converges to a constant value as $T_{R} \rightarrow 0$, thus we conclude that the cooling rate $\dot{T}_{R} \propto T_{R}^{2}$ is valid independent of the choice of $\delta$.

\section{DISCUSSION AND CONCLUSION}

We have shown that our natural modification of the model proposed by Cleuren et at. does not violate the dynamic version of the third law and allows for the same cooling performance at temperatures $T_{R}>\Delta$ as the original. This is a positive result, which tells us that the original model can be used to cool very efficiently down to the extreme limit of $T_{R} \sim \Delta$, where the

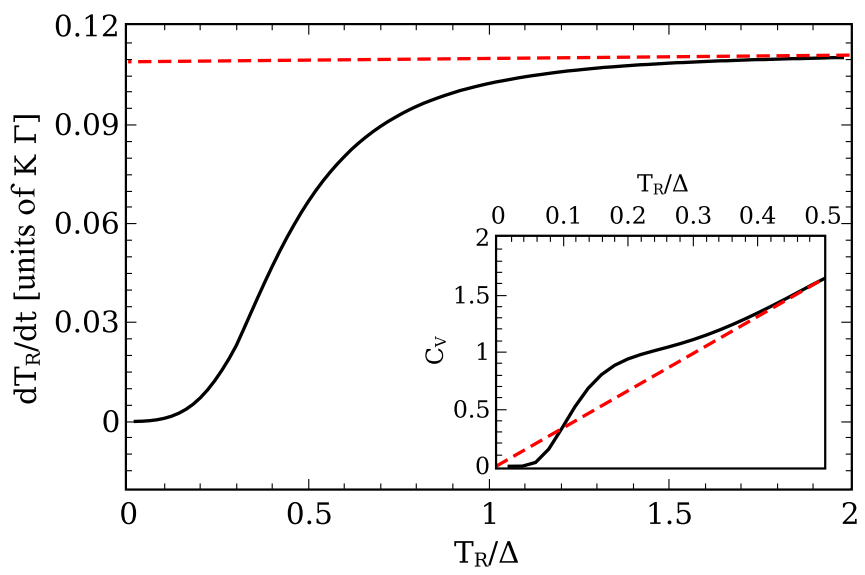

FIG. 5. Plot of the optimized cooling rate $d T_{R} / d t$ as a function of $T_{R} / \Delta$, Again we see that the discrete model (solid line) reproduces the third law violating constant rate of temperature change of the continuous model (dashed line) for $T_{R} \gtrsim \Delta$. The inset shows $C_{V}$ as a function of the same variable. When $T_{R} \lesssim \Delta / 2$ the heat capacity obtains a feature similar to the Schottky anomaly, indicating that the main contribution to the heat capacity comes from the two levels ( $\delta$ and $\delta-\Delta$ ) closest to $\mu=0$. As a result, for $T_{R} \lesssim \Delta$ the exponential term in $\dot{Q}_{R}$ cancels the one in $C_{V}$ and we are left with the $T_{R}^{2}$ term from the heat capacity. The parameters used are $\Gamma=\Gamma_{s}=1, T_{L}=20 \mathrm{~K}$, $T_{S}=6000 \mathrm{~K}, \epsilon_{g}=100 \mathrm{~K}, \Delta=1 \mathrm{~K}$, and $\delta=\Delta / 2$.

cooling power is quenched. Though we assumed a constant level spacing $\Delta$, the low-temperature behavior of the cooling rate is insensitive to this assumption since at $T_{R} \rightarrow 0$ only the two levels closest to the chemical potential are important.

The laws of thermodynamics are so general that they should apply to both classical and quantum systems. The third law in particular is a theory about the properties of a system as its temperature approaches absolute zero and at low temperatures quantum effects become important. Quantum theory predicts that confined systems have discretized energy levels and when the temperature $T$ becomes comparable to the spacing between energy levels $\Delta$, this discreteness needs to be taken into account. In [9] they use a continuous energy spectrum of the metal lead, disregarding the quantum discreteness. In the comments on the violation of the third law [10-13], they employ a heat capacity derived from quantum theory and it is this mixing of classical and quantum descriptions that leads to the breaking of the unattainability principle. If instead we use a pure classical expression for the heat capacity, which would be a constant as given by the equipartition principle, the unattainability principle would be satisfied [16].

One of the assumptions we have made is that the left hot lead functions as a large heat bath and has no effect on the cooling rate. A recent article [17] has shown that in a cooling process the density of states of the left heat bath affects the cooling rate of quantum refrigerators. A refined model where we take into account the properties of the left lead would give us additional insight into the nature of quantum refrigerators.

We have shown that the cooling power and cooling rate is quenched when $T \sim \Delta$. The energy-level spacing in metals is determined by the strength of confinement of the electrons. By increasing the volume of the lead that is to be cooled, the 
spacing $\Delta$ will decrease. Therefore, the temperature where the cooling power is quenched approaches absolute zero as the volume goes to infinity. However, by increasing the volume of the lead, the assumption of instantaneous equilibration of the electrons according to the Fermi-Dirac distribution becomes implausible; in reality, only a small area of the sample would be cooled and the lead would be in a nonequilibrium state. Larger leads also have a higher heat capacity and one must remove more heat per degree of temperature change than for smaller samples, which decreases the cooling efficiency. Finding the limit of efficient cooling for real systems by balancing these effects would be beneficial and relevant for future cooling technologies.

\section{ACKNOWLEDGMENTS}

The research leading to these results has received funding from the European Union Seventh Framework Program (FP7/2007-2013) under Grant Agreement No. 308850 (INFERNOS).
[1] J. Wang, Y. Lai, Z. Ye, J. He, Y. Ma, and Q. Liao, Phys. Rev. E 91, 050102 (2015).

[2] J.-H. Jiang, O. Entin-Wohlman, and Y. Imry, Phys. Rev. B 85, 075412 (2012).

[3] D. Venturelli, R. Fazio, and V. Giovannetti, Phys. Rev. Lett. 110, 256801 (2013).

[4] J. P. Palao, R. Kosloff, and J. M. Gordon, Phys. Rev. E 64, 056130 (2001).

[5] N. Linden, S. Popescu, and P. Skrzypczyk, Phys. Rev. Lett. 105, 130401 (2010).

[6] A. Levy and R. Kosloff, Phys. Rev. Lett. 108, 070604 (2012).

[7] U. Seifert, Rep. Prog. Phys. 75, 126001 (2012).

[8] R. Kosloff and A. Levy, Annu. Rev. Phys. Chem. 65, 365 (2014).

[9] B. Cleuren, B. Rutten, and C. Van den Broeck, Phys. Rev. Lett. 108, 120603 (2012).
[10] A. Levy, R. Alicki, and R. Kosloff, Phys. Rev. Lett. 109, 248901 (2012).

[11] B. Cleuren, B. Rutten, and C. Van den Broeck, Phys. Rev. Lett. 109, 248902 (2012).

[12] A. E. Allahverdyan, K. V. Hovhannisyan, and G. Mahler, Phys. Rev. Lett. 109, 248903 (2012).

[13] O. Entin-Wohlman and Y. Imry, Phys. Rev. Lett. 112, 048901 (2014).

[14] A. Levy, R. Alicki, and R. Kosloff, Phys. Rev. E 85, 061126 (2012).

[15] L. E. Reichl and I. Prigogine, A Modern Course in Statistical Physics (University of Texas Press, Austin, 1980), Vol. 71, Chap. 7.H.2.

[16] E. M. Loebl, J. Chem. Educ. 37, 361 (1960).

[17] L. Masanes and J. Oppenheim, arXiv:1412.3828. 